\title{
Quantification of Single-Molecule Protein Binding Kinetics in Complex Media with Prism-Coupled Plasmonic Scattering Imaging
}

Pengfei Zhang, ${ }^{\dagger}$ Guangzhong Ma, ${ }^{\dagger} Z_{i j i a n W a n}{ }^{\dagger, \neq}$ and ShaopengWang ${ }^{*, \dagger}$

${ }^{\dagger}$ Biodesign Center for Bioelectronics and Biosensors, Arizona State University, Tempe, Arizona 85287 United States

${ }^{\ddagger}$ School of Electrical, Energy and Computer Engineering, Arizona State University, Tempe, Arizona 85287, United States

*Email: Shaopeng.Wang@asu.edu 


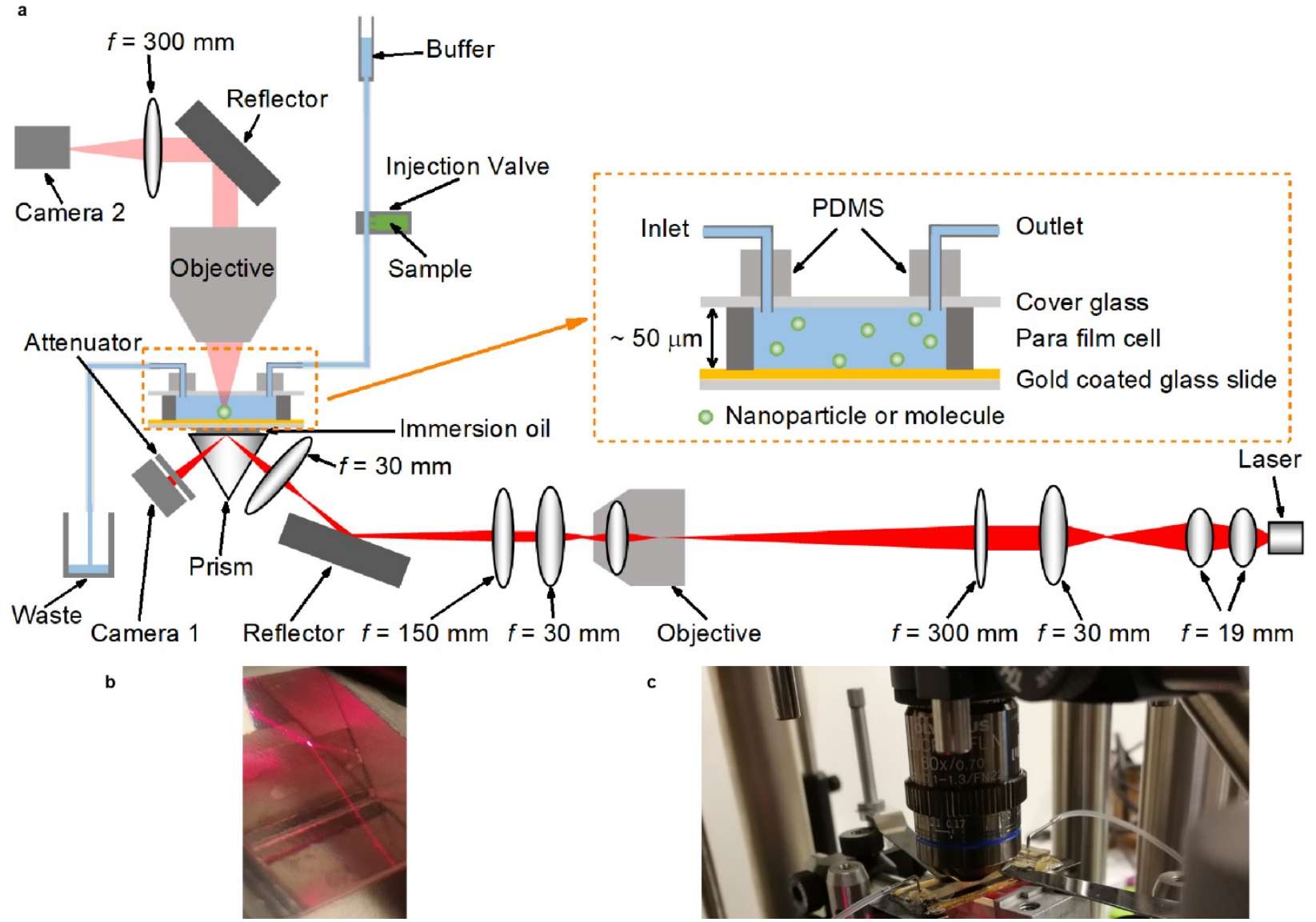

Figure S1 (a) Schematic diagram of the optical setup for prism based plasmonic scattering microscopy. Light from the laser is first conditioned by a pair of lenses with focal length of 19 $\mathrm{mm}$ and collimated with a $30 \mathrm{~mm}$ lens, and then focused to the back focal plane of a 100x objective by a tube lens with focal length of $300 \mathrm{~mm}$. Next, the Gaussian beam focused on the back focal plane of the objective was conditioned and directed on the prism surface with a lens group as shown in (b). The incident angle was adjusted by a manual translation stage to reach surface plasmon resonance (Thorlabs, Newton, NJ). Light reflected from the gold-coated glass slide is collected by a camera (Point Grey CM3-U3-13Y3M-CS) after an optical attenuator (ND40A, Thorlabs, Newton, NJ) to avoid overexposure, for helping to find the resonance angle. Scattered light from the protein and gold surface is collected by a top-mounted 60x objective (Olympus, LUCPLFLN60X, NA = 0.7) with a $300 \mathrm{~mm}$ tube lens for 100x magnification to form a PSM image on a second camera (MQ013MG-ON, XIMEA). The incident light intensity is $5 \mathrm{~kW} / \mathrm{cm}^{2}$ or less. A flow cell is designed for both sample delivery and liquid cooling to dissipate the heating effect of the incident light as described before. The gold-coated glass slide owns the size of $22 \mathrm{~mm} * 40$ $\mathrm{mm}$ to provide enough space for the objective as shown in subfigure (c). A gravity based liquid delivery system equipped with an injection valve is used to transport different samples onto the sensor surface. 


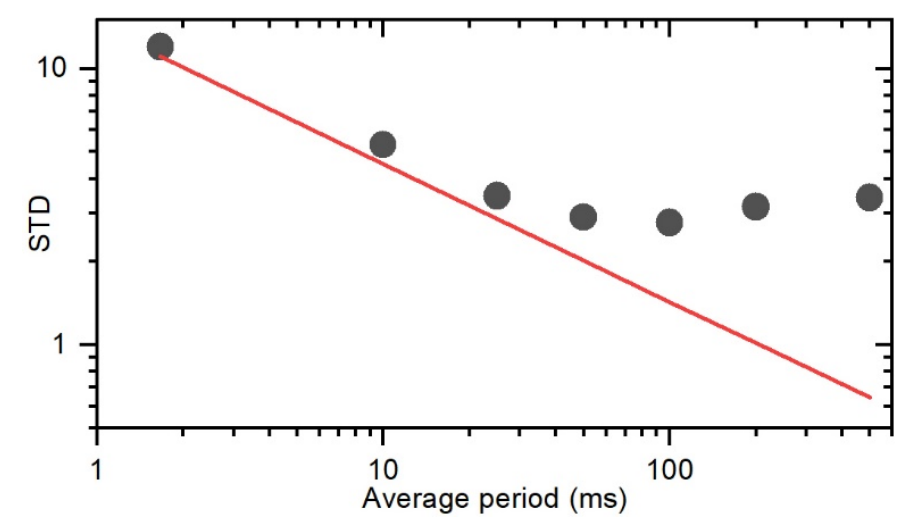

Figure S2. Standard deviation of background images recorded in the absence of analytes as a function of average period. The raw image sequence of a bare gold surface was recorded at 800 fps for $10 \mathrm{~s}$. Then, differential image sequences with different image average times were obtained. Next, total intensity of all pixels in a selected airy disk sized spot ( 18 pixels diameter circled area) for all images in the differential image sequences were calculated. Standard deviation of the total intensity in each $10 \mathrm{~s}$ differential image sequences are calculated and plotted as grey dots. The red line is expected shot noise value based on the total photons collected in the spot. Therefore, for the $50 \mathrm{~ms}$ or less averaging time used for the data analysis, shot noise is dominant. 

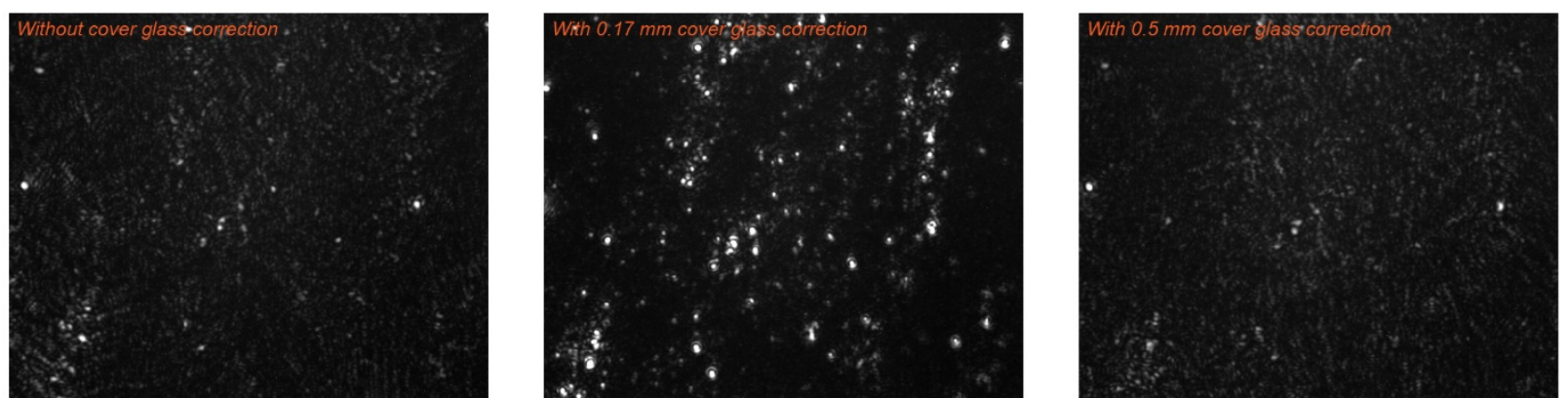

b

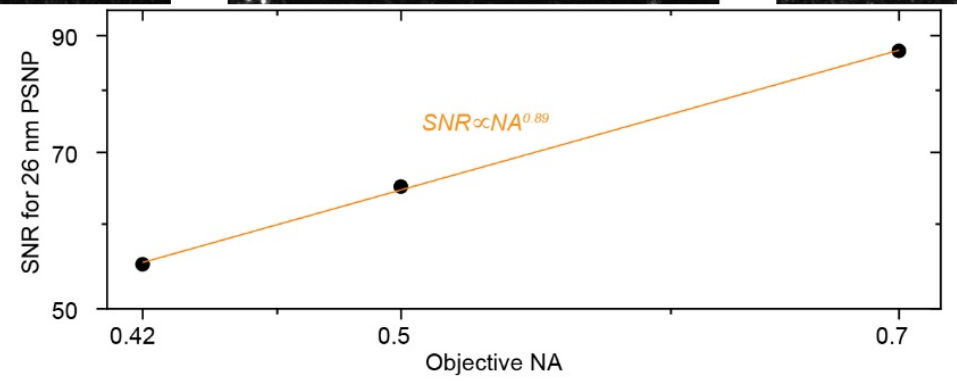

Figure S3. Effect of objective features on image quality. (a) Comparison of images from the same region using the $60 \mathrm{x}$ objective $(\mathrm{NA}=0.7$ ) with different cover glass correction parameters. (b) Signal noise ratio of standard $26 \mathrm{~nm}$ polystyrene nanoparticles (PSNP) detection against the numerical aperture (NA) of the collection objective. 

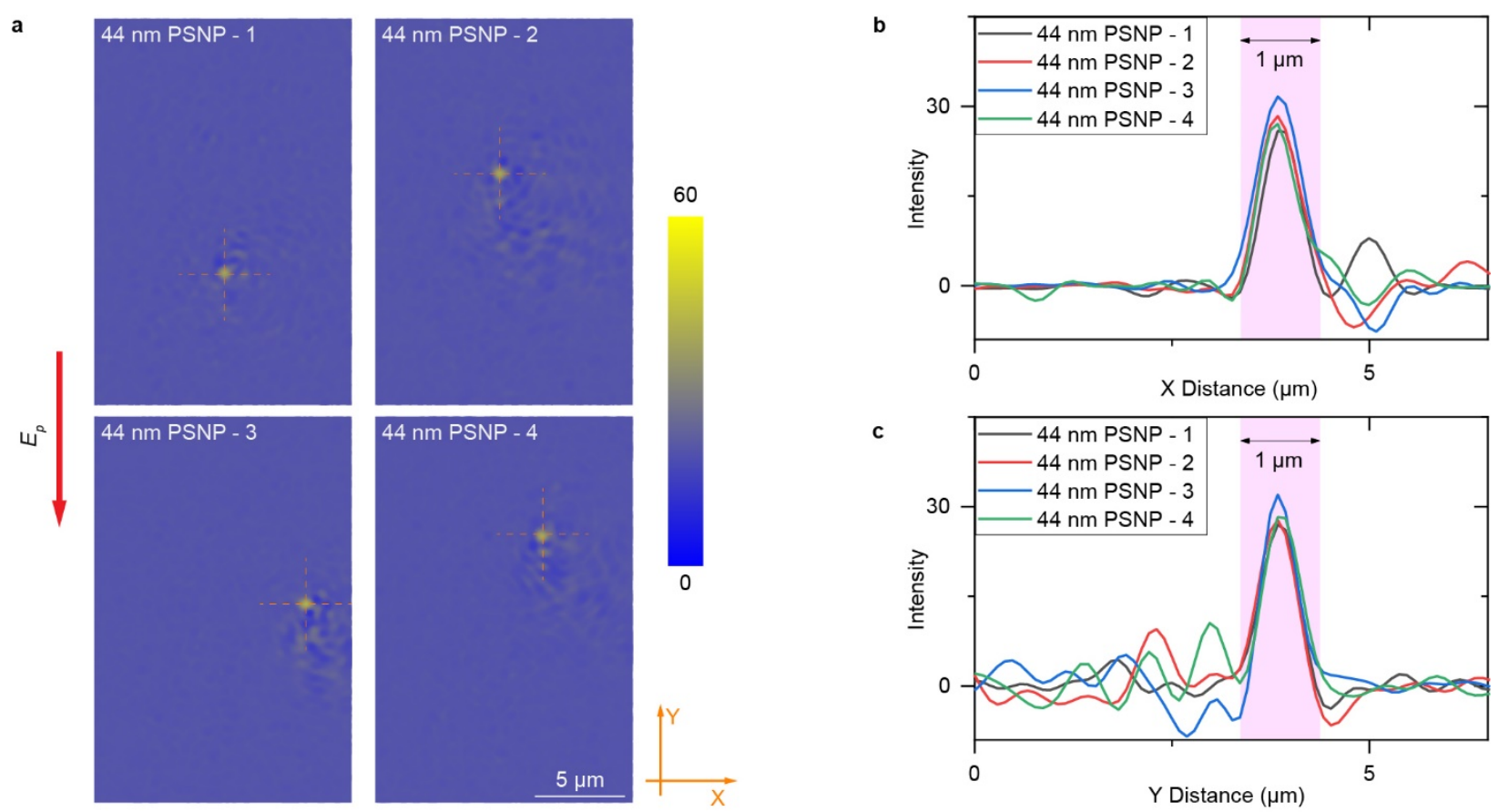

Figure S4 Delocalization feature of plasmonic scattering images. (a) PSM images of four individual $44 \mathrm{~nm}$ polystyrene nanoparticles at different locations. (b)- (c) Intensity profiles of the marked lines in (a). 

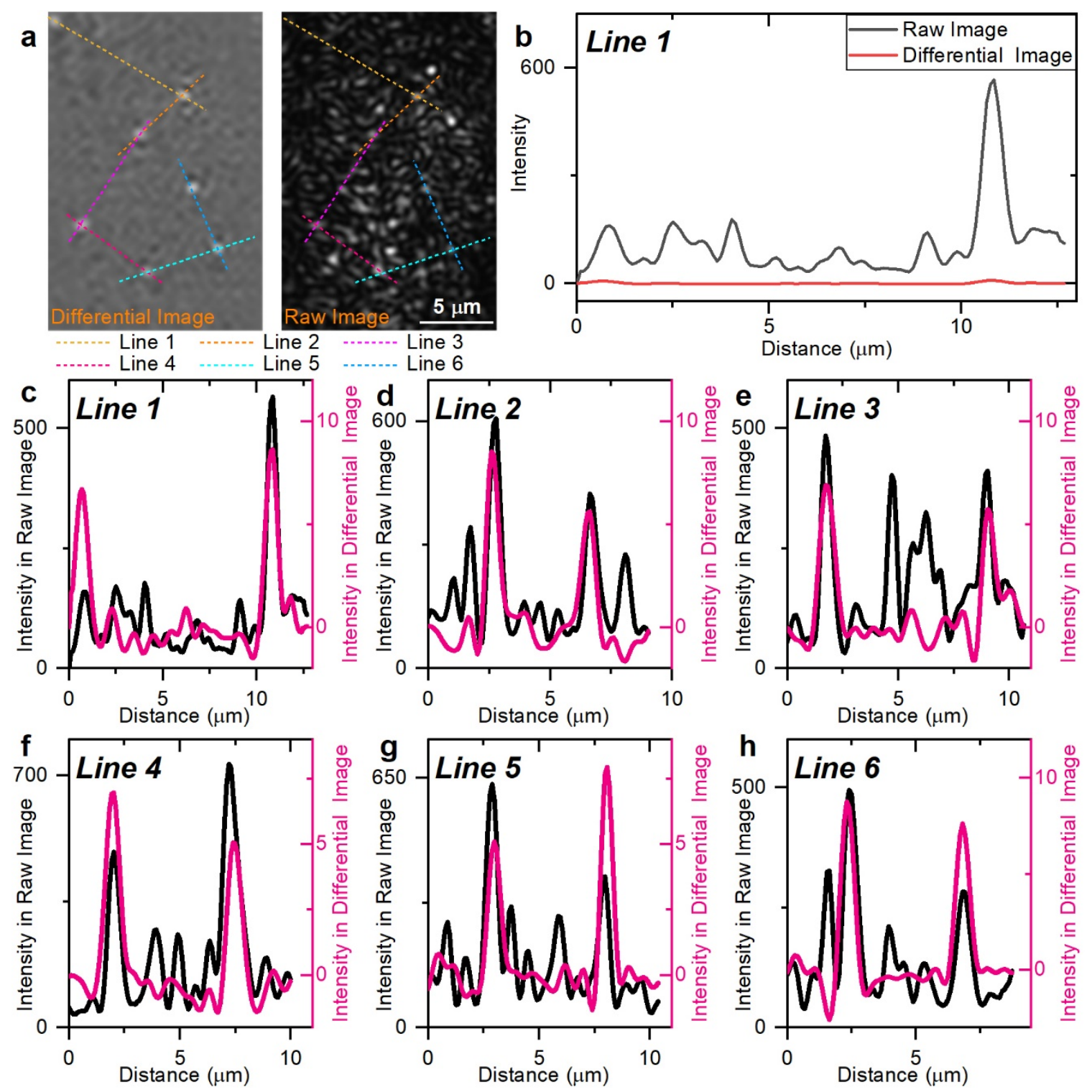

Figure S5. Comparison of line profiles in raw and differential PSM images. a) Raw and differential image of $26 \mathrm{~nm}$ polystyrene nanoparticles binding to the gold surface showing location of 6 selected lines. b) Raw and differential line profiles of Line 1 plotted in the same scale, which shows that the background scattering (raw profile) is much stronger than the $26 \mathrm{~nm}$ PS particle signal (differential profile). c-h) Raw and differential line profile plotted with different scale, which shows that the particle intensity is not depending on background intensity. Incident light intensity: $2.5 \mathrm{~kW} / \mathrm{cm}^{2}$. Exposure time: $2 \mathrm{~ms}$. Scale bars: $5 \mu \mathrm{m}$. 
a
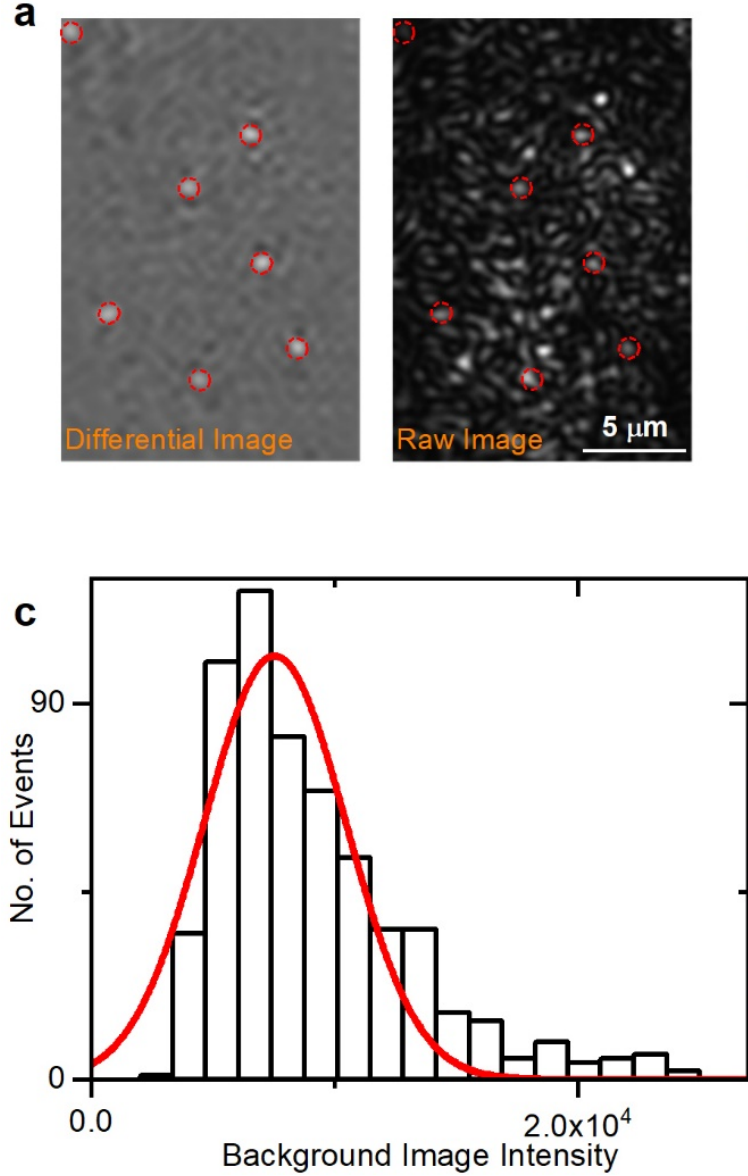
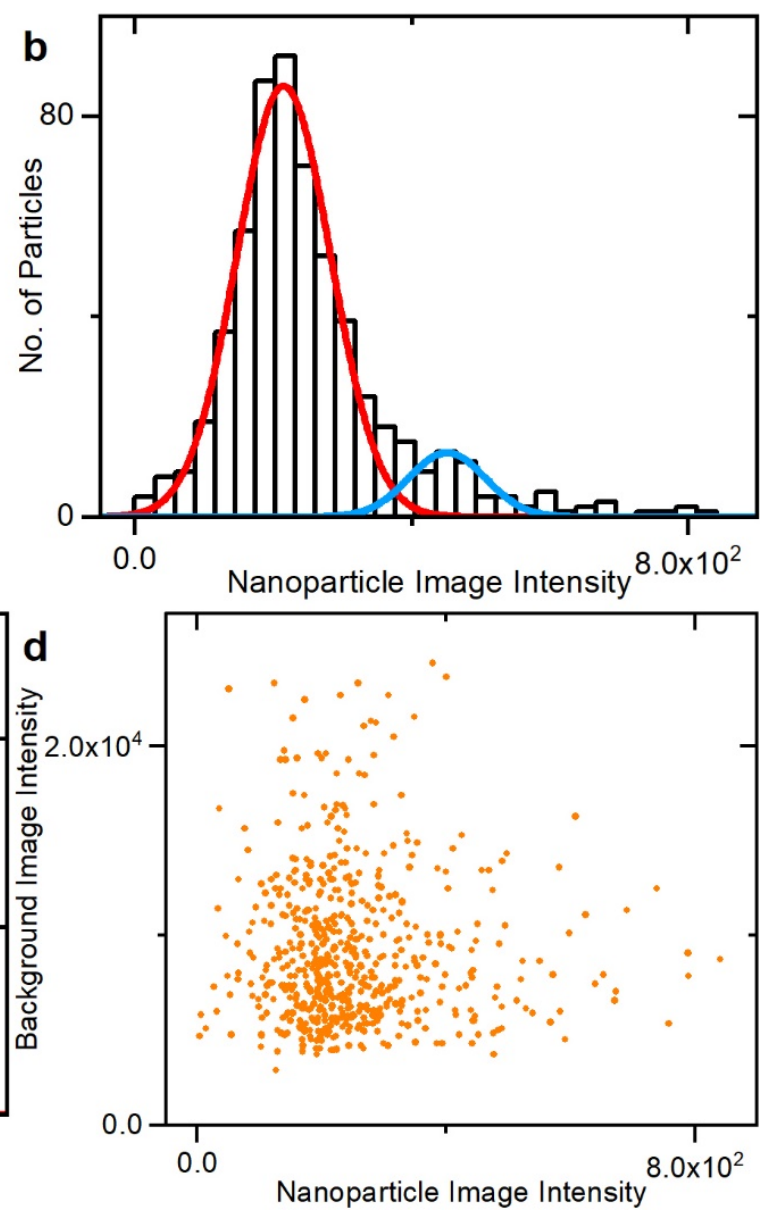

Figure S6. Comparison of image intensity at the nanoparticle binding position in raw and differential images. a) Raw and differential image of $26 \mathrm{~nm}$ polystyrene nanoparticles. b) Histogram of 590 nanoparticle image intensities extracted from differential images. The nanoparticle image intensity is total intensity of all pixels within the Airy disk centered around the nanoparticle binding location in differential image. The solid lines are Gaussian fittings. The small second peak (blue line) is attributed to formation of dimers or two particles binding to the nearby surface simultaneously within the diffraction limit. c) Histogram of corresponding background image intensity at the location of the 590 nanoparticles. The background image intensity is total intensity of all pixels within the Airy disk centered around the nanoparticle binding location in raw image. d) Correlation plot of nanoparticle image intensities versus background image intensities at the particle location. The correlation coefficient between nanoparticle and background image intensities is determined to be $\sim-0.0224$ with the corrcoef function in MATLAB, confirming that the PSM signal intensity is not depending on background scattering intensity. Incident light intensity: $2.5 \mathrm{~kW} / \mathrm{cm}^{2}$. Exposure time: $2 \mathrm{~ms}$. Scale bars: $5 \mu \mathrm{m}$. 

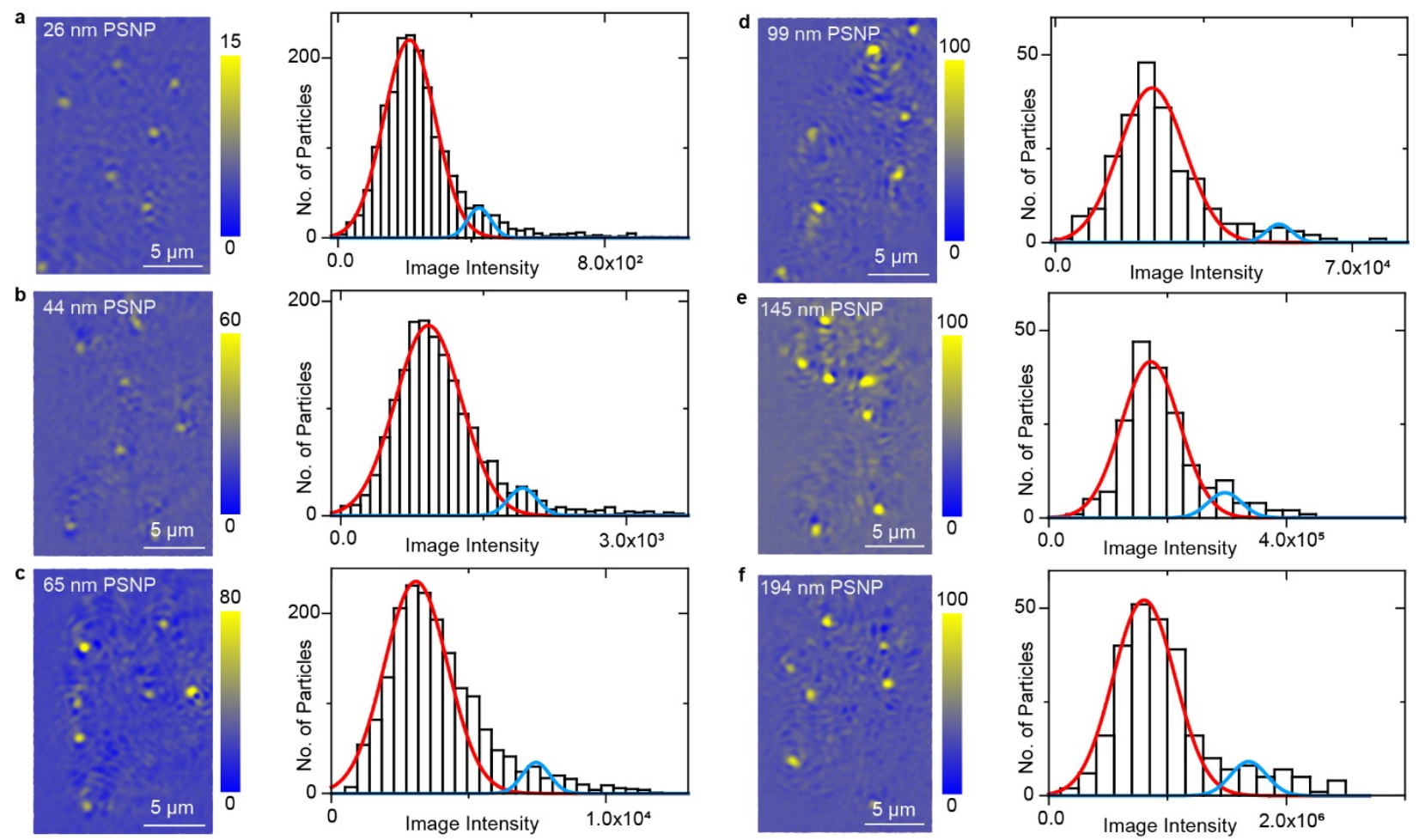

Figure S7. Calibration with polystyrene nanoparticles. (a-f) PSM images of $26 \mathrm{~nm}, 44 \mathrm{~nm}, 65$ $\mathrm{nm}, 99 \mathrm{~nm}, 145 \mathrm{~nm}$, and $194 \mathrm{~nm}$ polystyrene nanoparticles and corresponding image intensity histograms, where the solid lines are Gaussian fittings. The small secondary peak (blue) in each histogram is attributed to the formation of dimers or two particles binding to the nearby surface simultaneously within the diffraction limit. Incident light intensity and camera exposure time are $2.5 \mathrm{~kW} / \mathrm{cm}^{2}$ and $2 \mathrm{~ms}$ for $26 \mathrm{~nm}, 2.5 \mathrm{~kW} / \mathrm{cm}^{2}$ and $1 \mathrm{~ms}$ for $44 \mathrm{~nm}, 2.5 \mathrm{~kW} / \mathrm{cm}^{2}$ and $0.5 \mathrm{~ms}$ for 65 $\mathrm{nm}, 300 \mathrm{~W} / \mathrm{cm}^{2}$ and $2 \mathrm{~ms}$ for $99 \mathrm{~nm}, 300 \mathrm{~W} / \mathrm{cm}^{2}$ and $0.3 \mathrm{~ms}$ for $145 \mathrm{~nm}, 300 \mathrm{~W} / \mathrm{cm}^{2}$ and $0.03 \mathrm{~ms}$ for $194 \mathrm{~nm}$. The image intensity was normalized with incident light intensity of $2.5 \mathrm{~kW} / \mathrm{cm}^{2}$ and camera exposure time of $2 \mathrm{~ms}$. The histogram width results from the intensity fluctuations of binding events, which may be caused by the size and structure heterogeneities (PNAS 107 (37), 16028-16032 (2010); PNAS 115 (41), 10275-10280 (2018)). 


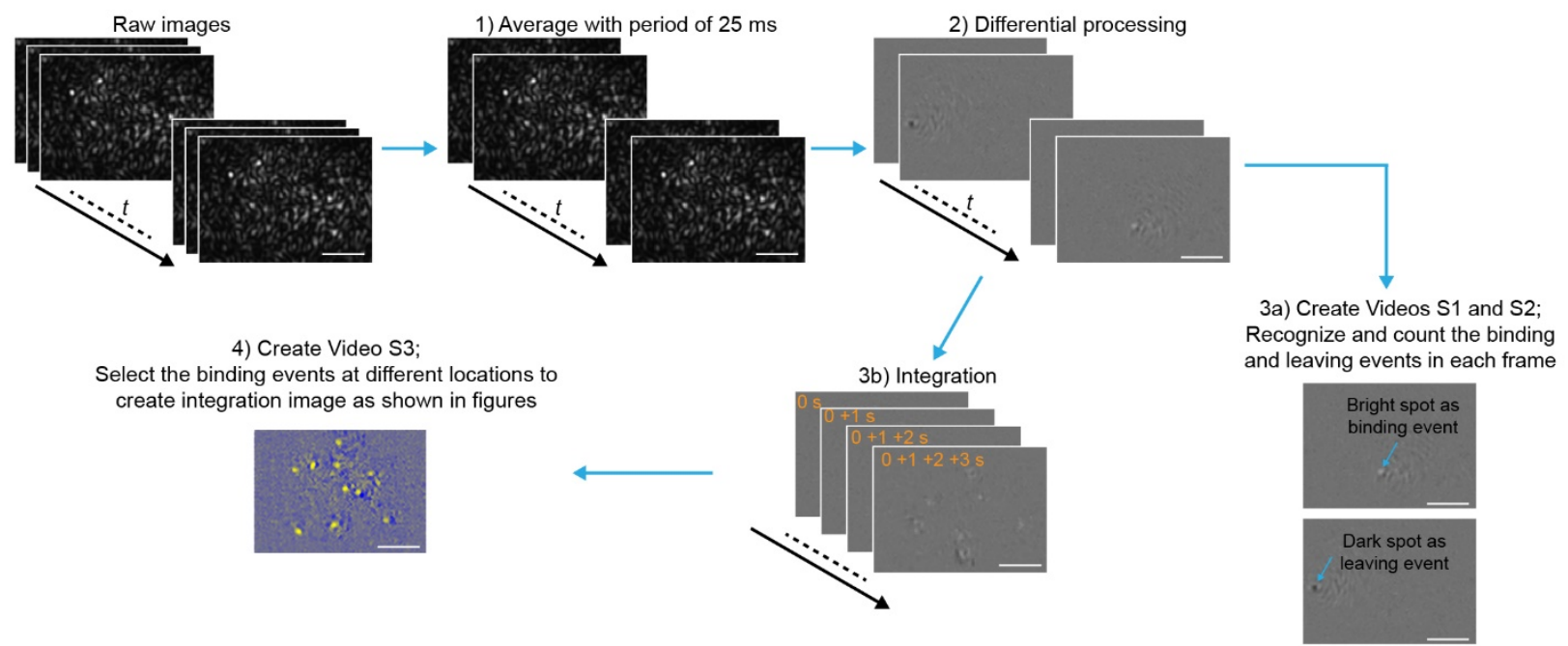

Figure S8. Image processing protocol. MATLAB and ImageJ source codes for the image processing have been provided before in Nature Methods 17, 1010-1017(2020). Scale bars: $5 \mu \mathrm{m}$. 


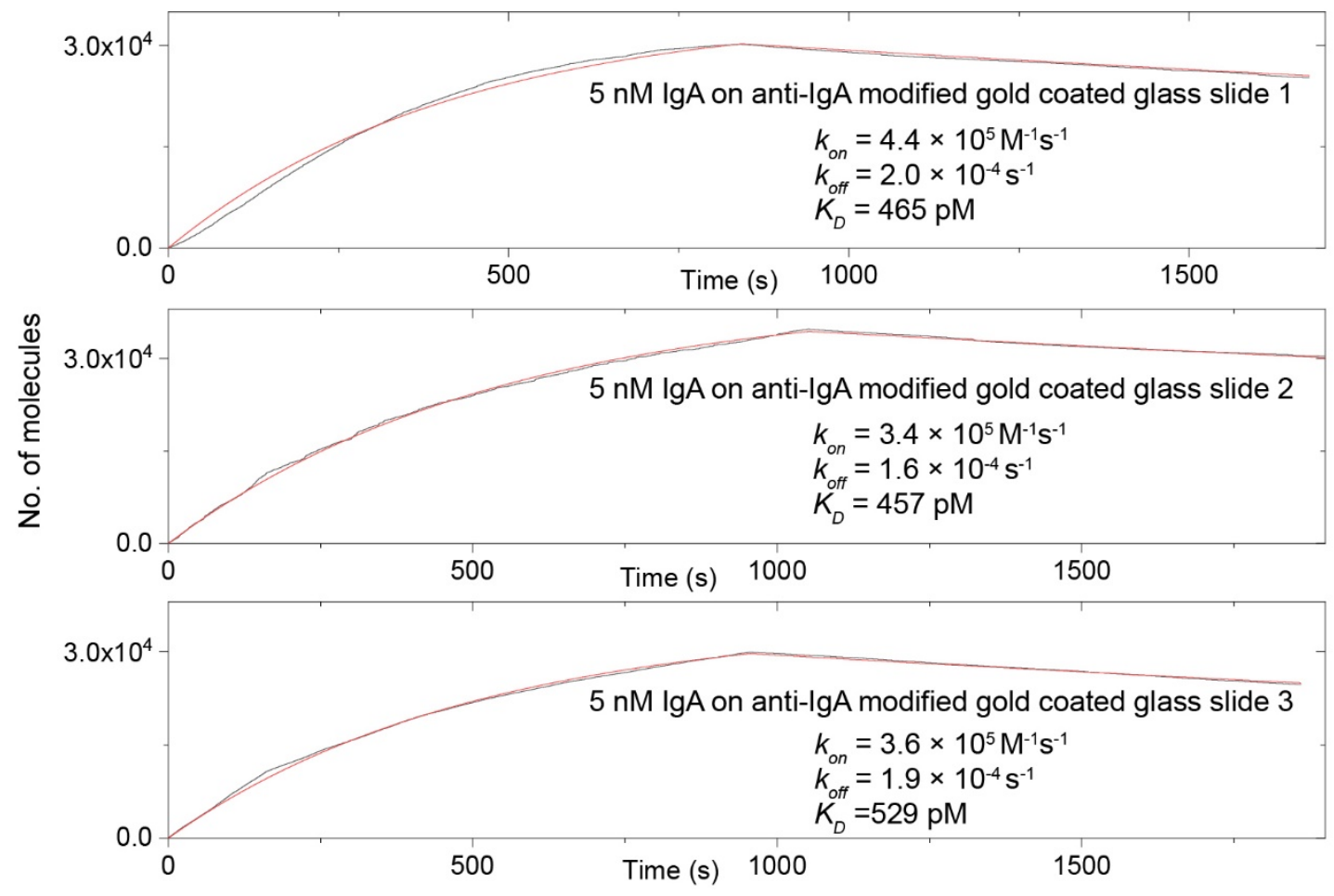

Figure S9. Kinetic response curves of IgA binding to anti-IgA determined by digitally counting of individual protein binding events on different gold coated glass slides. The association $\left(k_{o n}\right)$ and dissociation $\left(k_{o f f}\right)$ rate constants, and equilibrium dissociation constant $\left(K_{D}=k_{\text {off }} / k_{\text {on }}\right)$ are determined to be $3.8 \pm 0.5 \times 10^{5} \mathrm{M}^{-1} \mathrm{~s}^{-1}, 1.8 \pm 0.2 \times 10^{-4} \mathrm{~s}^{-1}$, and $484 \pm 39 \mathrm{pM}$, respectively. 


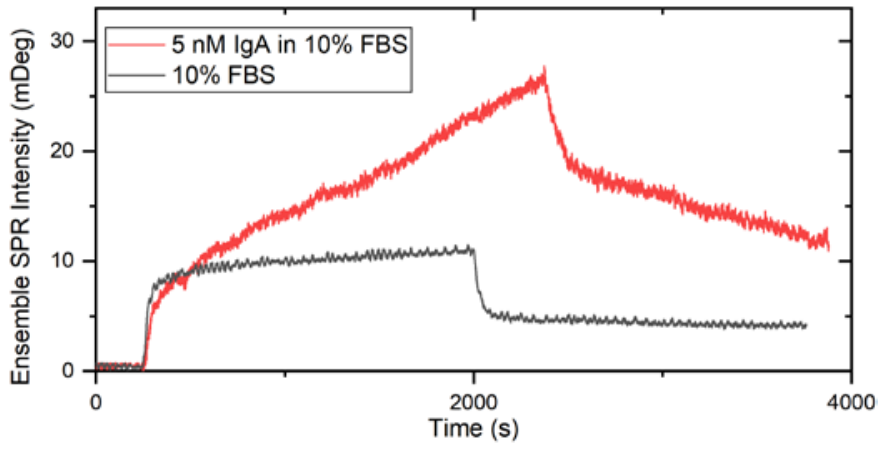

Figure S10. Ensemble SPR measurement results of flowing $10 \%$ fetal bovine serum (FBS, diluted with PBS buffer) with (red) and without (black) 5 nM IgA on anti-IgA modified gold. The baseline and dissociation phase are running with PBS buffer. The SPR intensity of 10\% FBS has a rapid jump in the beginning of association phase, caused by the bulk refractive index difference between 10\% FPS and PBS. Then, the SPR intensity of 10\% FPB has a small but steady increase for the rest of the association phase, which is due to non-specific binding of proteins in the FPS to the surface. After switching 10\% FBS back to PBS buffer, the beginning of dissociation phase also shows a rapid decrease in SPR intensity due to bulk refractive index shift, then a very slow decrease that indicating the slow dissociation of the non-specific bound proteins. The bulk refractive index shift and non-specific binding dramatically distorted the binding kinetic curve of IgA. 

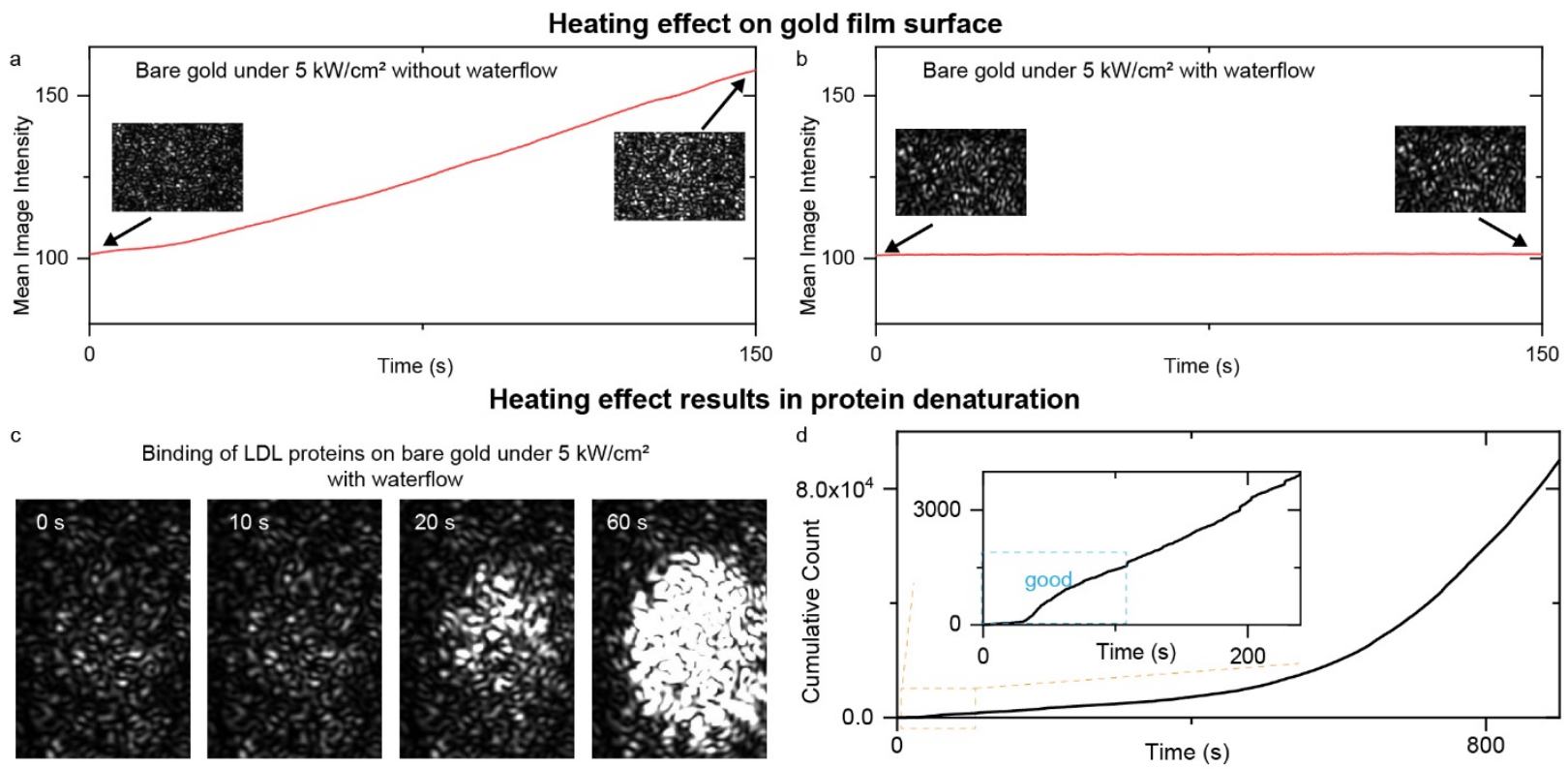

Figure S11. Heating effect on the PSM measurement performance.

Heating effect on gold film surface. (a) Add water on the gold coated glass slide without flowing under incident intensity of $5 \mathrm{~kW} / \mathrm{cm}^{2}$. The gold surface varies over time due to light heating, resulting unstable background. (b)Flowing water can effectively suppress the heating effect on gold surface.

Heating effect results in protein denaturation under high incident intensity. (c) Binding of LDL $\left(\mathrm{MW}=2300 \mathrm{kDa}\right.$ ) onto bare gold under incident intensity of $5 \mathrm{~kW} / \mathrm{cm}^{2}$ with flowing samples. While flowing water can remove the light induced heat and keep the proteins in solution safe, the bound proteins were likely damaged by the local heat on the gold surface and produced large intensity increase in a minute. This is the reason that incident intensity of $2.5 \mathrm{~kW} / \mathrm{cm}^{2}$ was employed for the detection of IgM and LDL proteins. (d) Binding of anti-BSA (IgG, MW = 150 $\mathrm{kDa}$ ) onto BSA modified gold measured with $5 \mathrm{~kW} / \mathrm{cm}^{2}$ incident intensity. Although the raw image intensity has no obvious variation as that in LDL protein binding process, the single molecule digital counting cannot work after $\sim 2$ minutes as the backgrounds in differential frames become noisy, resulting in the phenomenon that the counted binding curve obviously deviates from the first order binding kinetics. 

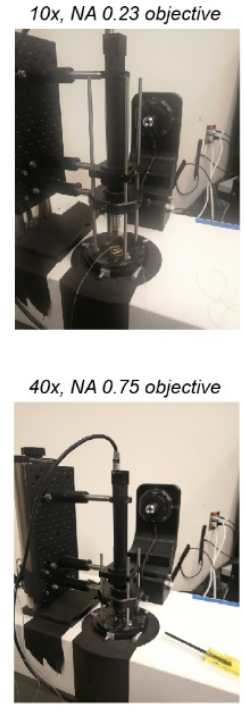
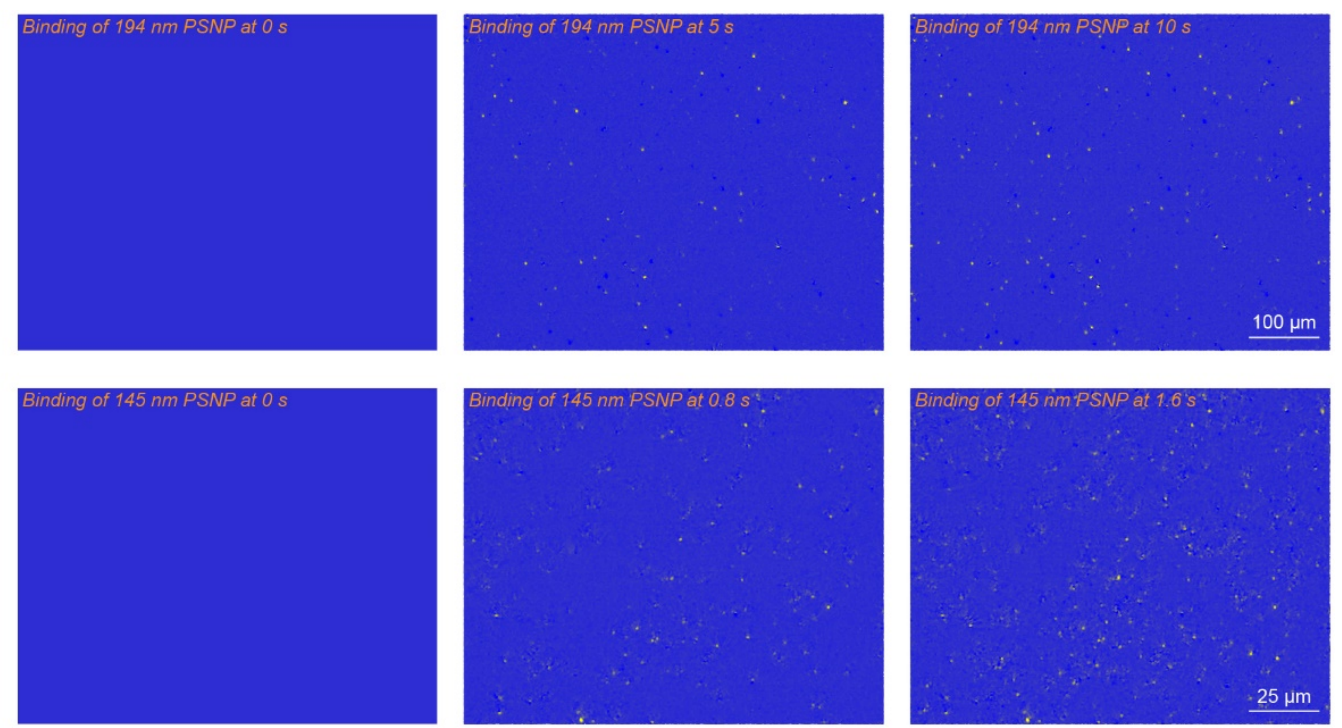

Figure S12. Implementation of PSM on a commercial SPRi device (SPRm 200, Biosensing Instrument Inc.). a) Using 10x objective (NA 0.23) as collection objective to image the binding process of individual $194 \mathrm{~nm}$ polystyrene nanoparticles (PSNP) on the bare gold. b) Using 40x objective (NA 0.75) as collection objective to image the binding process of individual $145 \mathrm{~nm}$ polystyrene nanoparticles (PSNP) on the bare gold. 


\section{Captions for Video files:}

Video S1, Dynamic binding events of individual nanoparticles on bare gold coated glass slide.

Video S2, Dynamic binding events of individual proteins on bare gold coated glass slide.

Video S3, Dynamic binding events of individual proteins on antibody modified gold coated glass slide. 